\title{
On the source of plastic flow in metallic glasses: Concepts and models
}

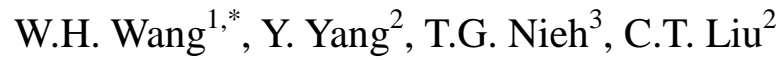 \\ ${ }^{1}$ Institute of Physics, Chinese Academy of Sciences, Beijing 100190, P. R. China \\ ${ }^{2}$ Centre for Advanced Structural Materials, Department of Mechanical and \\ Biomedical Engineering, City University of Hong Kong, Tat Chee Avenue, Kowloon \\ Tong, Kowloon, Hong Kong, P. R. China \\ ${ }^{3}$ Department of Materials Science \& Engineering, the University of Tennessee, \\ Knoxville, TN 37996-2200 \\ * corresponding author:whw@iphy.ac.cn
}

\begin{abstract}
We briefly review the state-of-the-art study on plastic flow in metallic glasses. Especially, we survey the features and behaviors, percolation, and response of the basic deformation units to the activation of stress and temperature, and various models and notions on microscopic flow in metallic glasses. The discussion, comments and perspective on possible unified notation, terminologies and models on plastic flow in metallic glasses are presented. The purpose is to reach a consensus within the community with a hope to eventually unify the notations and models on the deformations in metallic glasses.
\end{abstract}

Keywords: A. Metallic glasses; D. Plastic deformation unit; E. Yield behavior; B. Shear band; B. Plastic deformation mechanisms 


\section{Introduction}

Glassy state is a universal property of liquids if they are cooled rapidly enough, and is regarded as the fourth state of matter [1-5]. As a liquid is cooled, its atomic motions or dynamics slow down, resulting in a material just as solid as any crystal but without obvious long-range structural order of a crystal. Metallic glasses (MGs, also often referred to as amorphous alloys) are the simplest atomic glasses, which have spherical or nearly spherical constituents. Metallic glasses do not possess a microstructure in the traditional sense, where structural defects, such as dislocations, twins, stacking faults in a crystalline solid, are well defined and largely determine the mechanical and other properties of the solid. The atomistic mechanism of mechanical deformations, the atomic flow responded to stress or temperature, and failure in metallic glasses are very different from those in crystalline solids, and also different from the conventional picture [6]. In glasses, the medium-range topological heterogeneity in the order of only a few nanometers[7-9], is regarded as a result of density and/or compositional fluctuations, becomes an important parameter[7, 8].However, exact correlation between structural heterogeneity and resulting mechanical behavior remains mostly unresolved [7-9].

Studies have clearly demonstrated that in MGs, atomic processes in plastic flow is not so simply perceived[10]. Scientists both in material science and physics fields have made intensive efforts to elucidate the flow mechanism in MGs. Because plastic deformation of crystalline materials occurs due to motion of dislocations, search for similar "defects" in MGs has been attempted for long time. The current majority view on the phenomenon of flow, including mechanical failure and relaxations, and glass transition in MGs, appears to be that it is triggered by the activity of some structural "defects", such as free-volumes [11-13], shear transformation zones (STZs)[14-16], or soft liquid-like regions $\quad[17-20]$. The concentrations of such defects are assumed to be low, for instance, the free volume in a MG is only in the order of a percent in volume fraction $[15,17,21-23]$. Despite a small fraction, mechanical properties of the MGs depend sensitively on their "defects" [11, 15, 17, 21-34]. In crystalline solids, defects such as dislocations can be directly imaged using transmission electron 
microscopy, but such characterization techniques are considerably more difficult to apply to MGs in the absence of a long-range lattice. To find a direct evidence to correlate microscopic "defects" with plastic flow has proven to be extremely challenging. Macroscopically, MGs fail by forming well-defined shear bands. Many efforts were, therefore, made to study shear band formation. However, the shear banding process itself is very complicated and involves the interplay among temperature, local structural heterogeneity, free volume, and stresses. As a shear band propagates, it becomes intensively heated and, sometimes, melted by the dissipated energy of mechanical work [35], and this local melting essentially wipes out any structural change made by mechanical deformation. Consequently, it is almost impossible to relate the plastic deformation to the microstructure through shear bands.

There is yet another great challenge in discussing the flow behavior of metallic glasses in the community. Specifically, various terminologies/notations have been used to describe the onset of plasticity, plastic flow and fracture in metallic glasses. It often causes confusion and, sometimes, unnecessary dispute. Threfore, it might be useful to discuss the different terminologies and models at this stage. The purpose of the paper is, therefore, to summarize various notations/ terminologies and models on plastic flow in MGs with an attempt to compare and comment the physical assumptions behind these terminologies used in the description of different models. It may never be impossible to get a universal consensus within the community. However, a comparison of various models and notations/terminologies used in each model, the similarity and dissimilarity among them, and their mutual connections can certainly benefit the advance of the field.

\section{The notation, terminologies and models on plastic flow in MGs}

\subsection{Shear-transformation-zone modeling}

Classical theories that posit flow defects in MGs commonly refer to these as free volumes. As illustrated in Fig. 1(a), free-volume is defined as excess space among atoms which is necessary for atomic rearrangements in glassy solids[11-13]. The free-volume is probably the most widely used phenomenological concept in 
explaining flow, diffusion, structural relaxation, glass transition and mechanical properties. Free volumes facilitate atomic motion, including interatomic slips which would result in plastic deformation. However, the free-volume model was created for hard-sphere systems. In metallic materials, because of the harmonic nature of the interatomic potential, atoms can squeeze through tight space without free-volume when sufficient shear stress is applied. It was demonstrated that diffusivity in metallic liquids is surprisingly insensitive to pressure, whereas in the free-volume theory diffusivity is expected to be suppressed by pressure [36, 37]. Lewandowski have shown that the mechanical strength of metallic glasses is almost the same in tension and compression, and thus nearly independent of pressure [38], indicating that pressure has only minor effects. This result is consistent with the absence of the pressure effect on diffusion, and casts doubt on the free-volume mechanism for deformation. The free volume model has also been decisively challenged by diffusion studies, which demonstrated that atomic diffusion occurs in a collective manner rather than individual atom jumps as assumed by the free-volume theory [39, 40].

Alternatively, Argon [14] proposed the seminal concept of 'shear transformation zone' (STZ) in the late 1970s to explain plastic flow in metallic glass. Since then, extensive research interest has been stimulated in the field of metallic glasses, with an attempt to understand the nature and characteristics of STZs. Today, STZ may have become the most popular term used by us for explaining our experimental data; however, little attention has been paid to the fact that, indeed, there is more than one type of STZ models. In general, existing STZ models could be categorized into two types, with one picturing STZ as a "defect" and the source of plastic flow, and the other picturing STZ as an "event" and the consequence of plastic flow. The STZ model of Argon belongs to the latter type, according to which STZ is an event involving small collective atomic movements across tens of atoms $[14,16]$, as illustrated in Fig. 1(b). In the early development of the STZ model, it was assumed that these STZ events tend to occur in the regions of low atomic density [11, 12, 14]; however, through tracking the local intensive shear events in atomistic simulations, it was found that there is no deterministic correlation between local density and STZ 
event [41]. By comparison, STZs were ascribed to bond switching events in the atomic stress theory, which result from local topologic instability and can be triggered in both high and low density regions where unstable atomic configurations reside [42]. Here, it is worth mentioning that Johnson and Samwer [43] extended Argon's concept of STZ and merged it into the potential energy landscape perspective, and proposed a cooperative shear model to understand the deformation mechanisms and rheological properties of MGs. According to the cooperative shear model, activation of isolated STZs locally confined within an elastic matrix could be associated with the reversible fast $\beta$ relaxations, while percolation of these $\beta$ events leads to an irreversible collapse of the confining matrix and breakdown of elasticity that is associated with the slow $\alpha$ process. In such a sense, the mechanical response of MGs should be correlated with their relaxation behavior and the attributes of the underlying potential energy landscape. Namely, local hopping events initially occur in a small volume that releases a small amount of elastic energy showing a relatively smooth transition in the potential energy landscape; this subcritical processes continues until excessive energy accumulates, leading to unbalanced relaxation events and a shear catastrophe or plastic flow [43].

In contrast, the STZ model developed by Falk and Langer in the 1990s is based on the notion that STZ can be treated as pre-existing "flow defects" in metallic glasses, which comprise a group of atoms susceptible to structural rearrangements upon external agitations[44, 45]. In theory, the STZ defect is associated with a high effective temperature that is derived from high local configurational entropy [44, 45]. Conceptually, the concept of STZ defect proposed by Falk and Langer is similar to a variety of other notions discussed in the literature, such as flow units, liquid-like sites, movable regions, or mobile atoms, as will be discussed in the later text. The presence of these STZ defects may be rationalized from the perspective of structural dynamic heterogeneity in glassy solids, which comes about because of the non-equilibrium thermodynamics that governs glass transition in fragile glasses. For the theoretical treatment of the thermodynamic aspect of glass transition, interesting readers may refer to the random first order transition (RFOT) theory by Wolynes et al.[46] or the 
two-order-parameter model by Tanaka et al. [47, 48] for details. In what follows, let us call a STZ defect as a flow unit to avoid the possible confusion with a STZ event. Regardless of the physical nature of STZs, intensive work has been carried out to measure the sizes and activation energies of STZs. Based on the STZ models used, the results show that the activation barrier of STZs is roughly within the range of 30$80 \mathrm{~kJ} \mathrm{~mol}^{-1}$ and their activation volume approximately equal to $10 \AA^{3}[23,27,30]$.

\subsection{Thermal activation and mechanical relaxation}

According to the framework of the potential energy landscape (PEL) theory[49, 50], a glass-forming liquid system comprises a population of inherent states associated with local minima (basins) corresponding to the stable configurational states are separated by saddle points or energy barriers. The glass-forming alloy is supposed to locate at one of the local minima (or inherent states) in PEL. Figure 2 schematically illustrates the mechanism of plastic flow based on the PEL concept [51], and the inter-relation between STZ activation, plastic flow or yielding, and the $\beta$ - and $\alpha$-relaxations. Theoretically, the flow event or the configurational hopping in MGs is the process that the system escapes from one local minimum to another (an activated hopping between inherent states across energy barrier $\Delta E$ ) or the disappearance of barrier between neighbor local minima under stress. The $2 \mathrm{D}$ schematic illustration also shows the flow modes and their corresponding origin of potential energy landscapes: the $\beta$-mode is the stochastically and reversibly activated hopping events across "subbasins" confined within the inherent "megabasin" (intrabasin hopping) and the $\alpha$-mode is irreversible hopping events extending across different landscape megabasins (interbasin hopping). From the perspective of PEL, a potential nano-scale flow event, which is localized and confined by surrounding atoms, corresponds to the $\beta$-mode flow or $\beta$-relaxation; by contrast, the percolation of the flow units, entailing large scale atomic migration and irreversible structural change, leads to macroscopic yielding and plastic flow which corresponds to the $\alpha$-mode flow or $\alpha$-relaxation[51]. Recently, Wang et al.[52] further discovered a faster secondary relaxation ( $\beta$ ' relaxation) in a La-Ce-based metallic glass in addition to the 
conventional $\beta$-relaxation. This suggests that inelasticity in metallic glass may undergo three steps, with the process of initiation from the activation of the individual flow unit (fast $\beta$ ' relaxation), and transition to the "string-like" activation (slow $\beta$ relaxation) and finally to the percolation of the flow units that results in overall plasticity, as illustrated in Fig. 3. However, further research is still needed to confirm whether or not the fast $\beta$ ' relaxation is a universal process or specific to a certain type of metallic glass.

The activation energy of the flow in MGs is the principal source of information about their dynamics. The forced flow by sudden, rare and localized atomic or molecular rearrangement in $\mathrm{MG}$ is different from the flow in liquid, and it is non-Newtonian inhomogeneous flow. According to the elastic model $[5,43,51,53$, 54], the plastc deformation, glass transition, and relaxations in MGs can be treated as the flow phenomenon activated by different processes such as thermal process or mechanical process. The activation energy, $\Delta E$, barrier is a key factor for controlling the flow in both MGs and MG-forming liquids, and the activation energy has linear relationship with elastic moduli, $M[43,51,53,54]$, namely, $\Delta E \propto M$. The elastic model provides a simple picture for the flow in MGs and can clearly explain the time dependence (or strain dependence) of the plastic flow in MGs. From the perspective of flow defects, these defects are ubiquitous in MGs and occupy even as much as a quarter of the volume $[17,22,29,32,34,44]$. These concepts of defect are useful in describing the macroscopic and microscopic flows in MGs and the resultant variation in the local structure and physical properties is the principal feature of the glassy state, so that the local mechanical or dynamic or other physical responses also vary from place to place $[12,18,19,55-61]$.

In principle, one may consider the portions of the $\mathrm{MG}$ which respond strongly to the applied stress as defects even though direct experimental observation of such defects is difficult and may depend on how such defects are activated. Based on the random first-order transition theory, Xia and Wolynes [46] postulate that the flow in 
glasses are nucleated by liquid-like droplets characteristic of an incipient random first-order transition between solid-like and liquid-like (jammed and unjammed) phases. This postulate, plus some scaling analysis, produces the Vogel-Fulcher result. Similar concepts, such as the liquid-like sites from Cohen [62] and Egami [42, 63], were also proposed to rationalize the glass transition and plastic flow behavior of metallic glasses. For instance, Egami proposed that the liquid-like sites correspond to regions with the local volume strain $\varepsilon_{v}$ larger than $11 \%$, as a n-type free-volume $\left(\varepsilon_{v}>\right.$ 0.11 ) defect, and regions with the local volume strain $\varepsilon_{v}$ less than $-11 \%$, as the p-type anti-free-volume $\left(\varepsilon_{v}<-0.11\right)$ defect [62]. As such, the density of the liquid-like sites $p$ (liq), depends on temperature $T$, can be simply derived. It was estimated that the percolation concentration of the liquid-like sites for glass transition or flow in MGs be around 0.2 [62].

According to the random first order transition theory, the high mobility of the flow defect regions provides the structural features that enable the stress-driven change from thermally assisted viscous creep to superplastic flow [64-66]. Alternatively, Granato [22, 67, 68] proposed a model of interstitialcy "defect", which also leads to rather numerous successful interpretations of flow and relaxation phenomena in metallic glasses under different conditions [67-70]. Similar to the elastic models, the intersitialcy defect model predicts that the interstitialcy formation enthalpy $\Delta H$ is proportional to the shear modulus, $\Delta H=\alpha \Omega G,(\Omega$ is the volume per atom and $\alpha \approx 1)[67,68]$ and any change of the defect concentration will lead to heat release or heat absorption. This can be precisely monitored by measurements of the shear modulus because the latter is exponentially dependent on the defect concentration $c$ as: $G=G_{x} \exp (-\alpha \beta c)$, where $G_{x}$ is the shear modulus of the reference (maternal) crystal and $\beta$ is the dimensionless shear susceptibility.

To understand plasticity and glass transition in MGs, Wang et al. [59-61, 71-74] proposed dynamic flow units model, according to which MG can be regarded as the composite of perfect elastic matrix and liquid like flow units $(\mathrm{MG}=\mathrm{ideal}$ glass + flow units), and the flow phenomenon in MG is the percolation of the localized flow units. 
According to Wang et al [59-61, 71-74], the $\beta$-relaxation is the dynamic response of the atoms in the flow units which can be detected by dynamic spectrum like dynamic mechanical spectroscopy. The $\beta$-relaxation has the comparable activation energy with that of the flow unit and is closely related to the initiation and evolution of the flow units in MG. Consequently, the flow of MG is correlated with inherent relaxation processes. Thus, the distribution and evolution of energy barriers, size, and intrinsic relaxation time of flow units in a MG can be determined through the $\beta$-relaxation studies using an activation-relaxation method. One important outcome of the activation-relaxation experiments is the "universal" correlation $P(t)=\frac{P_{\infty}}{1+c}$, where $P(t)$ is some properties of MGs, $c$ the concentration of flow units, and $P_{\infty}$ is certain property of corresponding ideal glass. Such a correlation helps in understanding the evolution process of flow units, structural features and relaxation behaviors in MGs, which can also rationalize the effects of the cooling rate, aging, rejuvenation and annealing on properties and structure of metallic glasses. Meanwhile, it offers a compelling physical picture on the underlying structural origins of heterogeneities, deformation, relaxations and glass transition of metallic glasses [59-61, 71-74].

To quantitatively understand anelasticity in MGs, Liu and Yang recently proposed a 'core-shell' structural model [17, 57, 75-79] based on a similar physical picture that MGs comprise the nano-scale liquid-like regions trapped within an elastic glassy matrix. The 'core' region may be interpreted as the group of atoms exhibiting a lower packing density, a lower local modulus or a higher energy dissipation rate than those in the 'shell' region. In the core-shell model, the liquid-like cores may 'evolve' many times by changing their configurations, which, however, does not immediately cause the overall yielding of a MG if the elastic shells are still interconnected. Following the line of thinking, a dynamic structural evolution process can be pictured for MGs, entailing many sub-critical activation events in the apparent 'elastic' deformation regime and a mathematic model, taking into account the dynamic structural heterogeneity, is also developed, which enables one to quantitatively measure the physical/mechanical properties of the core-shell regions. 
Aside from the above general physical models, efforts were also made in elucidating the structural origin of the liquid-like sites or soft regions in a particular type of MGs. For instance, Ding and Ma [80] proposed that certain types of coordination polyhedra, which may be termed as those geometrically unfavored motifs (GUMs), constitute the most flexible local environments that encourage soft modes and high propensity for shear transformations in MGs[81]. On the other hand, Peng et al.[30] find that the degree of local five-fold symmetry (LFFS) can act as a structural indicator to predict the plastic deformation of the local structures and the plastic events prefer to be initiated in the regions with less degree of LFFS and propagate toward the region with more degree of LFFS. Their work is consistent with the longstanding notion that the liquid-like or soft sites are most likely associated with the "movable" regions with many possible configurations and therefore do not fit into any packing schemes, like crystalline-like or icosahedral packing, that could "geometrically" favor the stabilization of a glassy structure, which in turn serve as the "initiators" for inelasticity in metallic glasses. However, plastic flow is controlled by the breakdown of the LFFS, which constitute the geometrically favored structures or glass "stabilizers" that prevents irreversible structural change from occurring. In other words, the so-called GUM or the regions poor in LFFS is probably the structural origin for the onset of plasticity (or equivalently $\beta$-mode deformation) in metallic glasses.

\section{Summary and Outlook}

To summarize, different concepts and models are presented with a brief discussion in this short view article with regard to the basic "flow unit" in MGs. To facilitate understanding, Table I provides a short description of the major assumptions of these models and the phenomena that had be explained using each of them. Here, we should emphasize that the information provided in Table I only represents the view of the current authors, which is by no means a complete list. However, through this comparison, difference and similarity among different concepts and models can be 
conceived. Interestingly, it can be appreciated that many of the models were indeed developed more or less on a similar physical understanding, although the terminologies used are different and the mathematic formulation may vary because of different assumptions made. This indicates that a unified understanding is possible in future. In retrospect, the origin of plastic flow in glass is a very old topic in glass science but, till today, it has been remaining heavily debated in the community of materials science and condensed matter physics. With this brief review, we hope that some confusion and misunderstanding, if there is any, could be clarified and, more importantly, more research interest could be stimulated.

\section{Acknowledgements}

W.H.W appreciates the financial support of the NSF of China (Grant Nos. 51271195 and 51461165101) and the MOST 973 Program (No. 2015CB856800). Y.Y. acknowledges the financial support of the GRF fund provided by the Hong Kong Government (Grant No. CityU9042066). TGN is supported by NSF Contract DMR-0905979. 


\section{References}

[1] Y.T. Cheng, W.L. Johnson, Disordered materials: a survey of amorphous solids, Science, 235 (1987) 997-1002.

[2] F. Spaepen, The art and science of microstructural control, Science, 235 (1987) $1010-1014$.

[3] I.M. Hodge, Strong and fragile liquids-a brief critique, J. Non-Cryst. Solids, 202 (1996) 164-172.

[4] C.J. Byrne, M. Eldrup, Bulk metallic glasses, Science, 321 (2008) 502-503.

[5] J. Dyre, Mysteries of the glass transition, Phys. Today, 61 (2008) 15-15.

[6] M.W. Chen, Mechanical behavior of metallic glasses: Microscopic understanding of strength and ductility, Annu. Rev. Mater. Res., 38 (2008) 445-469.

[7] C. Bennemann, C. Donati, J. Baschnagel, S.C. Glotzer, Growing range of correlated motion in a polymer melt on cooling towards the glass transition, Nature, 399 (1999) 246-249.

[8] A. Furukawa, H. Tanaka, Inhomogeneous flow and fracture of glassy materials, Nat. Mater., 8 (2009) 601-609.

[9] K.D. Vargheese, A. Tandia, J.C. Mauro, Origin of dynamical heterogeneities in calcium aluminosilicate liquids, J. Chem. Phys., 132 (2010) 194501.

[10] L. Berthier, G. Biroli, J.P. Bouchaud, L. Cipelletti, D. El Masri, D. L'Hôte, F. Ladieu, M. Pierno, Direct experimental evidence of a growing length scale accompanying the glass transition, Science, 310 (2005) 1797-1800.

[11] F. Spaepen, A microscopic mechanism for steady state inhomogeneous flow in metallic glasses, Acta Metall., 25 (1977) 407-415. 
[12] P.S. Steif, F. Spaepen, J.W. Hutchinson, Strain localization in amorphous metals, Acta Metall., 30 (1982) 447-455.

[13] F. Spaepen, Homogeneous flow of metallic glasses: A free volume perspective, Scri. Mater., 54 (2006) 363-367.

[14] A.S. Argon, Plastic deformation in metallic glasses, Acta Metall., 27 (1979) 47-58.

[15] A.S. Argon, H.Y. Kuo, Plastic flow in a disordered bubble raft (an analog of a metallic glass), Mater. Sci. Eng., 39 (1979) 101-109.

[16] A.S. Argon, Strain avalanches in plasticity, Phil. Mag., 93 (2013) 3795-3808.

[17] J.C. Ye, J. Lu, C.T. Liu, Q. Wang, Y. Yang, Atomistic free-volume zones and inelastic deformation of metallic glasses, Nat. Mater., 9 (2010) 619-623.

[18] Y.H. Liu, D. Wang, K. Nakajima, W. Zhang, A. Hirata, T. Nishi, A. Inoue, M.W. Chen, Characterization of nanoscale mechanical heterogeneity in a metallic glass by dynamic force microscopy, Phys. Rev. Lett., 106 (2011) 125504.

[19] H. Wagner, D. Bedorf, S. Küchemann, M. Schwabe, B. Zhang, W. Arnold, K. Samwer, Local elastic properties of a metallic glass, Nat. Mater., 10 (2011) 439-442. [20] Z. Wang, P. Wen, L.S. Huo, H.Y. Bai, W.H. Wang, Signature of viscous flow units in apparent elastic regime of metallic glasses, Appl. Phys. Lett., 101 (2012) 121906.

[21] M.H. Cohen, D. Turnbull, Molecular transport in liquids and glasses, J. Chem. Phys., 31 (1959) 1164-1169.

[22] A.V. Granato, Interstitialcy model for condensed matter states of 
face-centered-cubic metals, Phys. Rev. Lett., 68 (1992) 974-977.

[23] Y. Shi, M.L. Falk, Strain localization and percolation of stable structure in amorphous solids, Phys. Rev. Lett., 95 (2005) 095502.

[24] S.G. Mayr, Activation energy of shear transformation zones: a key for understanding rheology of glasses and liquids, Phys. Rev. Lett., 97 (2006) 195501.

[25] F. Delogu, Identification and characterization of potential shear transformation zones in metallic glasses, Phys. Rev. Lett., 100 (2008) 255901.

[26] F. Delogu, Atomic mobility and strain localization in amorphous metals, Phys. Rev. Lett., 100 (2008) 075901.

[27] Y.Q. Cheng, A.J. Cao, E. Ma, Correlation between the elastic modulus and the intrinsic plastic behavior of metallic glasses: The roles of atomic configuration and alloy composition, Acta Mater., 57 (2009) 3253-3267.

[28] D. Rodney, C.A. Schuh, Distribution of thermally activated plastic events in a flowing glass, Phys. Rev. Lett., 102 (2009) 235503.

[29] W. Dmowski, T. Iwashita, C.P. Chuang, J. Almer, T. Egami, Elastic heterogeneity in metallic glasses, Phys. Rev. Lett., 105 (2010) 205502.

[30] H.L. Peng, M.Z. Li, W.H. Wang, Structural Signature of Plastic Deformation in Metallic Glasses, Phys. Rev. Lett., 106 (2011) 135503.

[31] S.T. Liu, Z. Wang, H.L. Peng, H.B. Yu, W.H. Wang, The activation energy and volume of flow units of metallic glasses, Scripta Mater, 67 (2012) 9-12.

[32] S.T. Liu, Z. Wang, H.L. Peng, H.B. Yu, W.H. Wang, The activation energy and volume of flow units of metallic glasses, Scripta Materialia, 67 (2012) 9-12. 
[33] L.S. Huo, J.F. Zeng, W.H. Wang, C.T. Liu, Y. Yang, The dependence of shear modulus on dynamic relaxation and evolution of local structural heterogeneity in a metallic glass, Acta Mater., 61 (2013) 4329-4338.

[34] S.T. Liu, W. Jiao, B.A. Sun, W.H. Wang, A quasi-phase perspective on flow units of glass transition and plastic flow in metallic glasses, J. Non-Cryst. Solids, 376 (2013) $76-80$.

[35] A.L. Greer, Y.Q. Cheng, E. Ma, Shear bands in metallic glasses, Mater. Sci. Eng. R, 74 (2013) 71-132.

[36] N.H. Nachtrieb, J. Petit, Self - Diffusion in Liquid Mercury, J. Chem. Phys., 24 (1956) 746-750.

[37] P. Klugkist, K. Rätzke, S. Rehders, P. Troche, F. Faupel, Activation Volume of C 57 o Diffusion in Amorphous Co 81 Zr 19, Phys. Rev. Lett., 80 (1998) 3288.

[38] R. Varadarajan, J.J. Lewandowski, Stress-State Effects on the Fracture of a Zr-Ti-Ni-Cu-Be Bulk Amorphous Alloy, Metall. Mater. Trans. A, 41 (2010) $1758-1766$.

[39] F. Faupel, W. Frank, M.P. Macht, H. Mehrer, V. Naundorf, K. Rätzke, H.R. Schober, S.K. Sharma, H. Teichler, Diffusion in metallic glasses and supercooled melts, Rev. Mod. Phys., 75 (2003) 237.

[40] W.H. Wang, H.Y. Bai, M. Zhang, J.H. Zhao, X.Y. Zhang, W.K. Wang, Interdiffusion in nanometer-scale multilayers investigated by in situ low-angle x-ray diffraction, Phys. Rev. B, 59 (1999) 10811.

[41] D. Srolovitz, V. Vitek, T. Egami, An atomistic study of deformation of 
amorphous metals, Acta Metall., 31 (1983) 335-352.

[42] T. Egami, Atomic level stresses, Prog. Mater. Sci., 56 (2011) 637-653.

[43] W.L. Johnson, K. Samwer, A Universal Criterion for Plastic Yielding of Metallic Glasses with a (T/Tg)2/3 Temperature Dependence, Phys. Rev. Lett., 95 (2005) 195501.

[44] M.L. Falk, J.S. Langer, Dynamics of viscoplastic deformation in amorphous solids, Phys. Rev. E, 57 (1998) 7192.

[45] J.S. Langer, Shear-transformation-zone theory of deformation in metallic glasses, Scri. Mater., 54 (2006) 375-379.

[46] X. Xia, P.G. Wolynes, Fragilities of liquids predicted from the random first order transition theory of glasses, Proc. Natl. Acad. Sci. USA, 97 (2000) 2990-2994.

[47] H. Tanaka, Two-order-parameter description of liquids. II. Criteria for vitrification and predictions of our model, J. Chem. Phys., 111 (1999) 3175-3182.

[48] H. Tanaka, Two-order-parameter description of liquids. I. A general model of glass transition covering its strong to fragile limit, J. Chem. Phys., 111 (1999) 3163-3174.

[49] M. Goldstein, Viscous liquids and the glass transition: a potential energy barrier picture, J. Chem. Phys., 51 (1969) 3728-3739.

[50] F.H. Stillinger, A topographic view of supercooled liquids and glass formation, Science, 267 (1995) 1935-1939.

[51] W.H. Wang, Correlation between relaxations and plastic deformation, and elastic model of flow in metallic glasses and glass-forming liquids, J. Appl. Phys., 110 (2011) 
053521.

[52] Q. Wang, S.T. Zhang, Y. Yang, Y.D. Dong, C.T. Liu, J. Lu, Unusual Fast Secondary Relaxation in Metallic Glass, Nat. Commun., 6 (2015) 7876.

[53] J.Q. Wang, W.H. Wang, Y.H. Liu, H.Y. Bai, Characterization of activation energy for flow in metallic glasses, Phys. Rev. B, 83 (2011) 012201.

[54] W.H. Wang, The elastic properties, elastic models and elastic perspectives of metallic glasses, Prog. Mater. Sci., 57 (2012) 487-656.

[55] T. Ichitsubo, E. Matsubara, T. Yamamoto, H.S. Chen, N. Nishiyama, J. Saida, K. Anazawa, Microstructure of Fragile Metallic Glasses Inferred from Ultrasound-Accelerated Crystallization in Pd-Based Metallic Glasses, Phys. Rev. Lett., 95 (2005) 245501.

[56] Y.H. Liu, G. Wang, R.J. Wang, D.Q. Zhao, M.X. Pan, W.H. Wang, Super plastic bulk metallic glasses at room temperature, Science, 315 (2007) 1385-1388.

[57] Y. Yang, J.F. Zeng, A. Volland, J.J. Blandin, S. Gravier, C.T. Liu, Fractal growth of the dense-packing phase in annealed metallic glass imaged by high-resolution atomic force microscopy, Acta Mater., 60 (2012) 5260-5272.

[58] P.Y. Huang, S. Kurasch, J.S. Alden, A. Shekhawat, A.A. Alemi, P.L. McEuen, J.P. Sethna, U. Kaiser, D.A. Muller, Imaging atomic rearrangements in two-dimensional silica glass: Watching silica's dance, Science, 342 (2013) 224-227.

[59] W. Jiao, P. Wen, H.L. Peng, H.Y. Bai, B.A. Sun, W.H. Wang, Evolution of structural and dynamic heterogeneities and activation energy distribution of deformation units in metallic glass, Appl. Phys. Lett., 102 (2013) 101903. 
[60] Z.G. Zhu, P. Wen, D.P. Wang, R.J. Xue, D.Q. Zhao, W.H. Wang, Characterization of flow units in metallic glass through structural relaxations, J. Appl. Phys., 114 (2013) 083512.

[61] Z. Wang, B.A. Sun, H.Y. Bai, W.H. Wang, Evolution of hidden localized flow during glass-to-liquid transition in metallic glass, Nat. Commun., 5 (2014) 5823.

[62] M.H. Cohen, G.S. Grest, Liquid-glass transition, a free-volume approach, Phys. Rev. B, 20 (1979) 1077.

[63] Y. Suzuki, T. Egami, Shear deformation of glassy metals: Breakdown of Cauchy relationship and anelasticity, J. Non-Cryst. Solids, 75 (1985) 361-366.

[64] J.P. Garrahan, D. Chandler, Coarse-grained microscopic model of glass formers, Proc. Natl. Acad. Sci. USA, 100 (2003) 9710-9714.

[65] J.S. Langer, A. Lemaître, Dynamic model of super-Arrhenius relaxation rates in glassy materials, Phys. Rev. Lett., 94 (2005) 175701.

[66] A. Wisitsorasak, P.G. Wolynes, On the strength of glasses, Proceedings of the National Academy of Sciences, 109 (2012) 16068-16072.

[67] A.V. Granato, D.M. Joncich, V.A. Khonik, Melting, thermal expansion, and the Lindemann rule for elemental substances, Appl. Phys. Lett., 97 (2010) 171911.

[68] A.V. Granato, Interstitialcy theory of simple condensed matter, Eur. Phys. J., 87 (2014) 1-6.

[69] V.A. Khonik, N.P. Kobelev, Alternative understanding for the enthalpy vs volume change upon structural relaxation of metallic glasses, J. Appl. Phys., 115 (2014) 093510. 
[70] A.N. Tsyplakov, Y.P. Mitrofanov, A.S. Makarov, G.V. Afonin, V.A. Khonik, Determination of the activation energy spectrum of structural relaxation in metallic glasses using calorimetric and shear modulus relaxation data, J. Appl. Phys., 116 (2014) 123507.

[71] H.B. Yu, W.H. Wang, H.Y. Bai, Y. Wu, M.W. Chen, Relating activation of shear transformation zones to $\beta$ relaxations in metallic glasses, Phys. Rev. B, 81 (2010) 220201(R).

[72] H.B. Yu, X. Shen, Z. Wang, L. Gu, W.H. Wang, H.Y. Bai, Tensile Plasticity in Metallic Glasses with Pronounced $\beta$ Relaxations, Phys. Rev. Lett., 108 (2012) 015504.

[73] W. Jiao, B.A. Sun, P. Wen, H.Y. Bai, Q.P. Kong, W.H. Wang, Crossover from stochastic activation to cooperative motions of shear transformation zones in metallic glasses, Appl. Phys. Lett., 103 (2013) 081904.

[74] Z. Lu, W. Jiao, W.H. Wang, H.Y. Bai, Flow unit perspective on room temperature homogeneous plastic deformation in metallic glasses, Phys. Rev. Lett., 113 (2014) 045501.

[75] Y. Yang, J.F. Zeng, J.C. Ye, J. Lu, Structural inhomogeneity and anelastic deformation in metallic glasses revealed by spherical nanoindentation, Appl. Phys. Lett., 97 (2010) 261905.

[76] Z.Y. Liu, Y. Yang, A mean-field model for anelastic deformation in metallic-glasses, Intermetallics, 26 (2012) 86-90.

[77] Z.Y. Liu, Y. Yang, C.T. Liu, Yielding and shear banding of metallic glasses, Acta 
Mater., 61 (2013) 5928-5936.

[78] Z.Y. Liu, M.W. Chen, C.T. Liu, Y. Yang, Origin of yielding in metallic glass: Stress-induced flow, Appl. Phys. Lett., 104 (2014) 251901.

[79] B.A. Sun, Z.Y. Liu, Y. Yang, C.T. Liu, Delayed shear banding and evolution of local plastic flow in a metallic glass, Appl. Phys. Lett., 105 (2014) 091904.

[80] J. Ding, Y.Q. Cheng, E. Ma, Correlating local structure with inhomogeneous elastic deformation in a metallic glass, Appl. Phys. Lett., 101 (2012) 121917.

[81] J. Ding, S. Patinet, M.L. Falk, Y.Q. Cheng, E. Ma, Soft spots and their structural signature in a metallic glass, Proc. Natl. Acad. Sci. USA, 111 (2014) 14052-14056. 


\section{List of Figure Captions}

Figure 1. The schematic illustration of (a) the free-volume model and (b) the shear-transformation-zone model.

Figure 2. A 2D schematically illustration of STZ activation (top left panel), $\alpha$-relaxation (flow and yielding, top right panel), and their corresponding origination of potential energy landscapes (bottom panel). Filled circles represent atoms with low propensity of motion, while open circles represent atoms with high propensity of motion. The arrows indicate the possible motion of atoms. The potential STZ events are localized with cooperative nature and is reversible due to confinements of surrounding materials, while $\alpha$-relaxation (percolation of STZs, or plastic flow and yielding) incorporates large scale atomic migration and irreversible. Below: A schematically illustration of flow and its corresponding origination of potential energy landscapes. There are two kinds of flow modes: the $\beta$-mode is the stochastically and reversibly activated hopping events across "subbasins" confined within the inherent "megabasin" (intrabasin hopping) and the $\alpha$ mode is irreversible hopping events extending across different landscape megabasins (interbasin hopping) [51].

Figure 3. The relaxation spectrum obtained from a La-based MG showing the primary $(\alpha)$ relaxation, slow and fast secondary $(\beta)$ relaxations, suggestive of a three-step inelastic deformation mechanism in MGs. (Data taken from Ref.[52]) 


\section{List of Table Captions}

Table I. Comparison of the different models and theories for plastic flow and mechanical relaxation in MGs and glassy solids. 


\section{List of Figures}

(a)
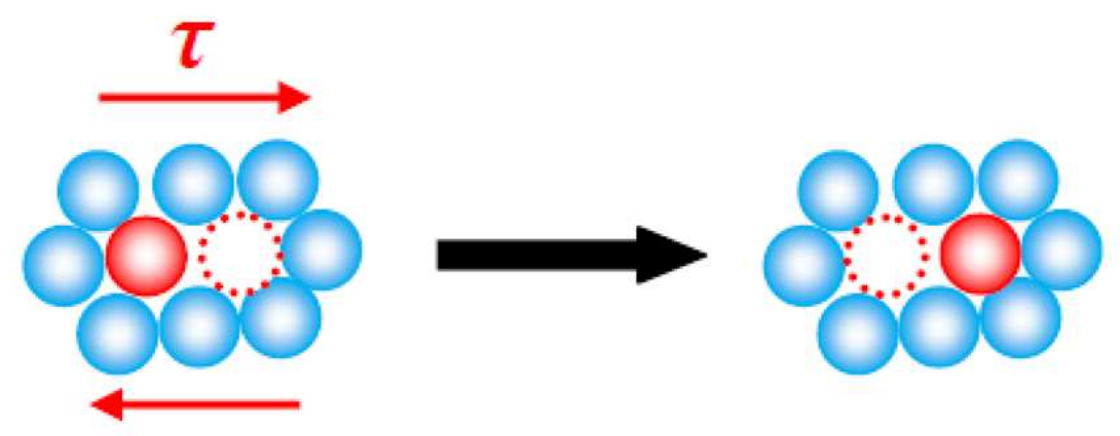

(b)
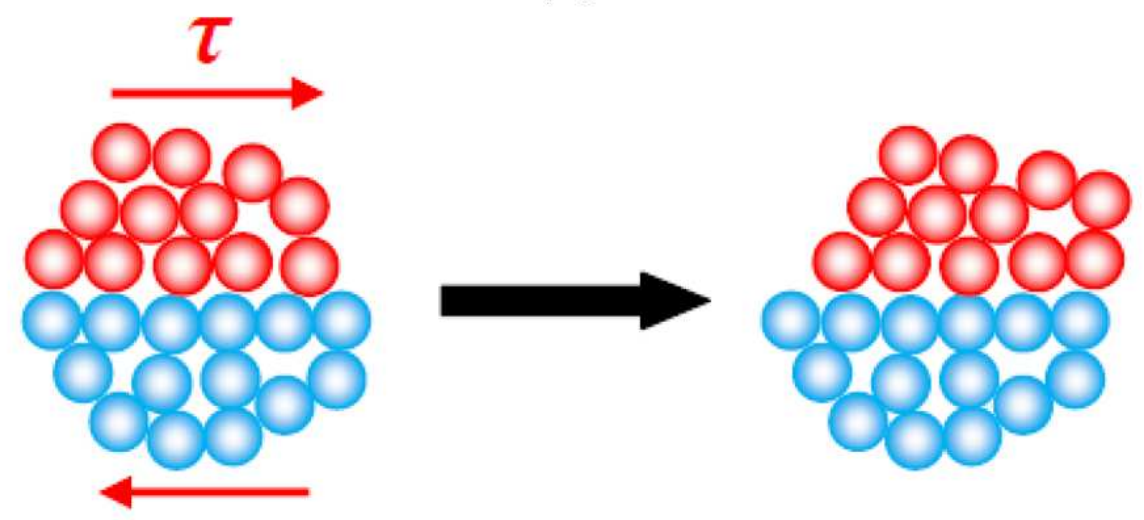

Figure 1 Wang et al. 


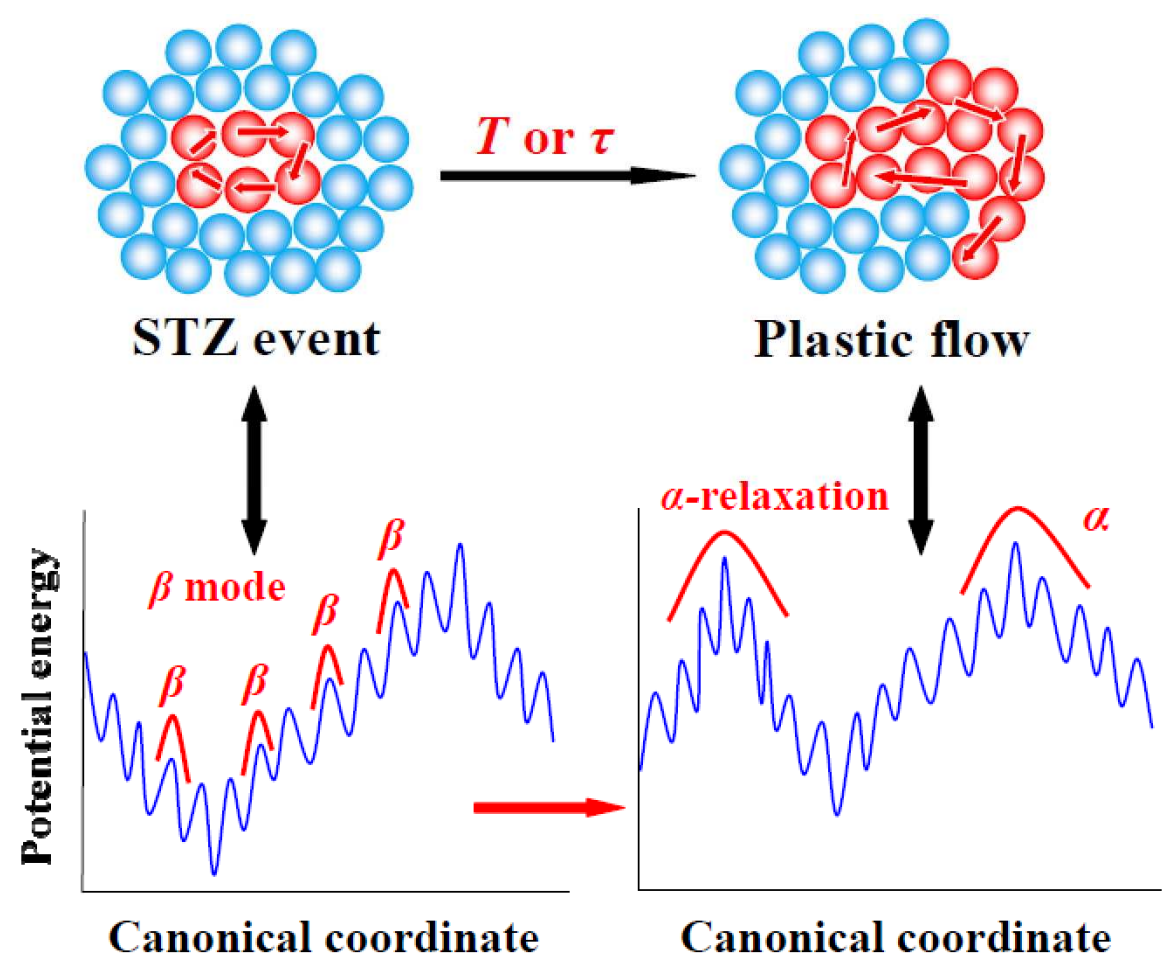

Figure 2 Wang et al. 


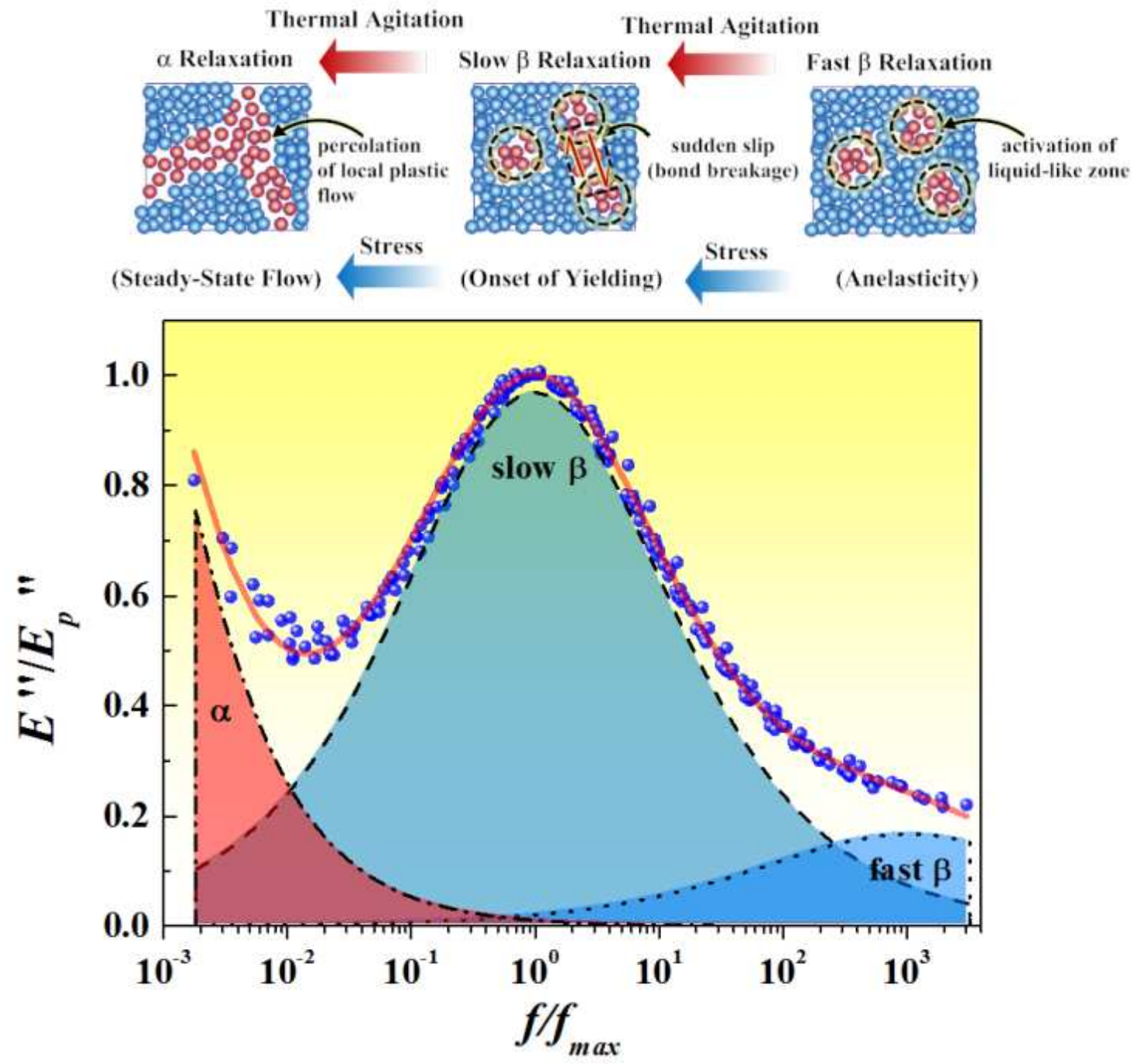

Figure 3 Wang et al. 


\section{List of Tables}

\begin{tabular}{|c|c|c|}
\hline Type & Major Assumptions & Phenomena Explained \\
\hline $\begin{array}{l}\text { Free-Volume } \\
\text { Model[11-13] }\end{array}$ & $\begin{array}{l}\text { Excess space exsits among atoms which } \\
\text { is necessary for structural } \\
\text { rearrangements through atom jump. }\end{array}$ & $\begin{array}{l}\text { "Transport" Properties, such as } \\
\text { diffusion, and Plastic Flow }\end{array}$ \\
\hline $\begin{array}{l}\text { Liquid Droplet } \\
\text { Model [21, 62] }\end{array}$ & $\begin{array}{l}\text { Glass comprises nano-scale liquid-like } \\
\text { droplets as a result of fast quenching. }\end{array}$ & Glass Transition \\
\hline $\begin{array}{l}\text { The STZ Model of } \\
\text { A. Argon [14-16] }\end{array}$ & $\begin{array}{l}\text { STZ is pictured as an "event" and the } \\
\text { consequence of platic flow. Plasticity } \\
\text { occurs once STZ events take place in } \\
\text { low-density regions. }\end{array}$ & Plastic Flow \\
\hline $\begin{array}{c}\text { The STZ Model of } \\
\text { Falk and Langer } \\
{[44,65]}\end{array}$ & $\begin{array}{l}\text { STZ is a flow defect that triggers plastic } \\
\text { flow in MGs, which is physically a local } \\
\text { region associated with high effective } \\
\text { temperature }\end{array}$ & Platic Flow and Rheology \\
\hline $\begin{array}{l}\text { Cooperative Shear } \\
\text { Model (CSM) [43] }\end{array}$ & $\begin{array}{l}\text { Plastic flow is a instability process that } \\
\text { mathematically conforms to a fold } \\
\text { catastrophe and microscopically results } \\
\text { from the percolation of the reversible } \\
\text { STZ events that can be associated with } \beta \\
\text { relaxation. }\end{array}$ & Plastic Flow and Rheology \\
\hline $\begin{array}{l}\text { Atomic Stress } \\
\text { Theory }[42,63]\end{array}$ & $\begin{array}{l}\text { Plastic flow is a instability process and } \\
\text { results from bond switching events, } \\
\text { which tend to occur in "free-volume" } \\
\text { and "anti-free-volume" regions. }\end{array}$ & $\begin{array}{c}\text { Glass Transition, Anelasticity } \\
\text { and Plastic Flow }\end{array}$ \\
\hline $\begin{array}{c}\text { Core-Shell } \\
\text { Model }[17,57,75, \\
76]\end{array}$ & $\begin{array}{l}\text { MGs are composed of liquid-like sites } \\
\text { (core) embedded into an elastic glassy } \\
\text { matrix (shell). }\end{array}$ & $\begin{array}{c}\text { Anelasticity, Mechanical } \\
\text { Relaxation and Glassy } \\
\text { Rheology }\end{array}$ \\
\hline $\begin{array}{c}\text { Interstitialcy } \\
\text { theory }[22,67,68]\end{array}$ & $\begin{array}{l}\text { It considers the intersticalcies as the } \\
\text { structural defects for glassy solids. }\end{array}$ & $\begin{array}{c}\text { Plastic Flow and Mechanical } \\
\text { Relaxation }\end{array}$ \\
\hline $\begin{array}{c}\text { Random } \\
\text { First-Order Phase } \\
\text { Transition } \\
\text { Theory }[46,66]\end{array}$ & $\begin{array}{l}\text { A glassy structure is dynamically } \\
\text { heterogeneous, consisting of regions of } \\
\text { different relaxation times }\end{array}$ & $\begin{array}{c}\text { Glass Transition, Plastic Flow } \\
\text { and Mechanical Relexation }\end{array}$ \\
\hline $\begin{array}{c}\text { Flow Unit } \\
\text { Model[59-61, } \\
71-74]\end{array}$ & $\begin{array}{l}\text { The MG is a composite of elastic matrix } \\
\text { and liquidlike flow units, and the flow } \\
\text { phenomenon is the peecolation of } \\
\text { localized flow units. } \beta \text {-relaxation is the } \\
\text { dynamic response of the atoms in the } \\
\text { flow units. }\end{array}$ & $\begin{array}{c}\text { Plastic Flow and Mechanical } \\
\text { Relaxation }\end{array}$ \\
\hline
\end{tabular}

